\title{
HISTOPATHOLOGICAL SPECTRUM OF VARIOUS BIRADS CATEGORIES OF BREAST LESIONS- A RETROSPECTIVE STUDY.
}

\section{Dr. P. Ramana Kumari* \\ Dr. M. Harika \\ Dr. G.Jahnavi \\ Dr. G. Saila Bala}

MD, Associate Professor, Department of Pathology, Siddhartha Medical College, Vijayawada, Andhra Pradesh, India. *Corresponding Author

MBBS,Post graduate, Department of Pathology, Siddhartha Medical College, Vijayawada, Andhra Pradesh, India.

DMRD DNB,Chief Radiologist, Jahnavi Imaging Centre, Guntur, Andhra Pradesh, India.

MD, Professor \& HOD, Department of Pathology, Siddhartha Medical College, Vijayawada, Andhra Pradesh, India.

ABSTRACT Introduction: Carcinoma of breast is the most common cancer of women, the incidence of which continue to increase worldwide. Sonomammography is a sensitive method for the diagnosis of breast cancer

preoperatively.

Aim: The aim of the present study is to evaluate the histopathological findings of various categories of BIRADS breast lesions and to analyse the discordant cases.

Materials and methods: This is a retrospective study of patients who have undergone mammography and simultaneous breast core needle biopsy. This study includes a total of 252 cases.

Results: Of these cases 2/252(0.8\%) cases were assigned BIRADs category II, 16/252(6.4\%) were assigned BIRADs category III, $64 / 252(25.4 \%)$ cases were assigned as BIRADs category IV and 170/252 (67.4\%) were assigned as BIRADs category V. Conclusion: In this study Radio-pathological concordance has been observed in $88 \%$ cases.

\section{KEYWORDS : BIRADS, histopathology, breast, carcinoma, sonomammography}

\section{INTRODUCTION}

Carcinoma of breast has become the most common cancer of women, the incidence of which continue to increase worldwide. Preoperative diagnosis constitutes an essential part of the workup of breast lesions. Histopathological examination remains the gold standard for diagnosing breast carcinoma.

Mammography is cost efficient and widely accepted technique to evaluate clinically suspected breast lesions and also for screening of breast cancer. High resolution sonography is a useful adjunct modality and helps characterizing a mammographically non-detected palpable abnormality, especially dense breast.

The American College of Radiology developed the Breast imaging, Reporting and Data System (BIRADS) to standardise the terminology employed for mammographic reports elaborately. The fourth BIRADS edition of November 2003 proposed seven categories for mammographic findings.[1]

0 . Incomplete, need additional imaging

1. Negative for malignancy

2. Benign

3. Probably benign

4. Suspicious of malignancy

5. Highly Suggestive of malignancy

6. Known Biopsy-Proven Malignancy.

The category 4 was subdivided into subcategories because of broad, compatible findings ranging from DCIS to Invasive Breast cancer. Subcategories include 4A(Low suspicion for malignancy), $4 \mathrm{~B}$ (Moderate Suspicion for malignancy) and $4 \mathrm{C}$ (High suspicion for malignancy). The percentages of cases diagnosed with breast cancer are $2-9 \%$ in category $4 \mathrm{~A}, 10-49 \%$ in $4 \mathrm{~B}$ and $50-94 \%$ in $4 \mathrm{C}$, indicating that some benign breast lesions are initially wrongly interpreted as highly suspicious of malignancy. $[2,3]$

The aim of the present study is to evaluate the histopathological findings of various categories of BIRADS breast lesions and to analyse the discordant cases.

\section{MATERIALS AND METHODS}

This is a retrospective study of patients who have undergone mammography and simultaneous breast core needle biopsy. This study includes a total of 252 cases.

The inclusion criteria of present study are BIRADS categories $2,3,4,4 \mathrm{~A}, \mathrm{~B}, \mathrm{C}$ and 5 on sonomammography, with corresponding core needle biopsy.

The exclusion criteria of the present study are male breast lesions, BIRADS categories 0, 1, inadequate BIRADS grading and cases without corresponding core biopsy specimen.

\section{Imaging study Protocol}

Imaging study including ultrasound and mammography was performed in 2 views ( craniocaudal and mediolateral oblique views) and grouped into BIRADS categories.

\section{Biopsy protocol}

Core Needle Biopsy was performed by the same radiologist, who performed the ultrasound and 3 cores were obtained from each patient and were submitted for histopathological examination. The core biopsy specimens were fixed in $10 \%$ buffered formalin, routinely processed through graded alcohols, cleared in xylol, impregnated and embedded in paraffin. 4 um thick sections were cut and stained with haematoxylin and eosin (H\&E) and examined by a pathologist using a light microscope.

The histopathological findings were correlated with BIRADS categories.

The cases with discordance between BIRADS category and histopathological findings are analysed and discussed.

\section{RESULTS}

A total of 252 cases of various BIRADS category breast lesions are included in the study.

In the present study, the age distribution of the patients ranged from 26 years to 89 years. The mean age was 53.6 years. 
Majority of the cases were recorded in 6th decade, ie 51 to 60 years, constituting $27.7 \%$. (Ref Table 1 )

Table 1: showing Age distribution of the total 252 cases, of various BIRADs category of breast lesions.

\begin{tabular}{|l|l|l|}
\hline Age group & No of cases & \% of cases \\
\hline $21-30$ & 9 & 3.7 \\
\hline $31-40$ & 57 & 22.7 \\
\hline $41-50$ & 64 & 25.3 \\
\hline $51-60$ & 70 & 27.7 \\
\hline $61-70$ & 38 & 15.1 \\
\hline $71-80$ & 12 & 4.7 \\
\hline $81-90$ & 2 & 0.8 \\
\hline Total & 252 & 100 \\
\hline
\end{tabular}

Out of total 252 cases, 2 cases $(0.8 \%)$ were of BIRADs category II, 16 cases $(6.4 \%)$ were of BIRADs category III, 64 cases (25.4\%) were of BIRADS category IV and 170 cases $(67.4 \%)$ of BIRADS category V( Ref Table 2).

Out of the total 64 cases of BIRADS category 4, only 27 were subcategorised as $4 \mathrm{~A}, 4 \mathrm{~B}, 4 \mathrm{C}$ ie, 10 cases were grouped as $4 \mathrm{~A}$, 5 cases as $4 \mathrm{~B}$ and 12 cases as $4 \mathrm{C}$.

Table 2 : Showing Distribution of various BIRADS category of breast lesions.

\begin{tabular}{|l|l|l|}
\hline BIRADS category & No of cases & $\%$ of cases \\
\hline II & 2 & $0.8 \%$ \\
\hline III & 16 & $6.5 \%$ \\
\hline IV & 37 & $25.4 \%$ \\
IVa & 10 & \\
IVb & 05 & \\
IVc & 12 & \\
\hline V & 170 & $67.4 \%$ \\
\hline Total & 252 & $100 \%$ \\
\hline
\end{tabular}

BIRADs Category V lesions (Highly suggestive of malignancy):

Out of 170 cases, assigned BIRADS cat V , 165 (97\%)cases showed histological features of carcinoma of breast- NOS type. Discordance with histopathological findings was recorded in 5 cases, in which 1 case showed histological features of Phyllodes tumour, 1 case of granulomatous mastitis and 3 cases showed histological features of papilloma.

BIRADs Category IV lesions (Suspicious of malignancy aLow suspicion, b-Moderate suspicion, c- High Suspicion): A total of 64 cases were recorded as BIRADs category IV, ie suspicious of malignancy.

Out of the total 64 cases of BIRADS category IV, only 27 were subcategorised as $4 a, 4 b, 4 c$ ie, 10 cases were grouped as $4 a$, 5 cases as $4 \mathrm{~b}$ and 12 cases as $4 \mathrm{c}$.

And on histopathological examination 46(72\%) cases showed features of malignancy, majority invasive carcinoma of breast-NST, l case each of mucinous carcinoma and poorly differentiated carcinoma.

The remaining 18(28\%) cases showed ie- 6 cases of Category IV, 6 cases of IVa, 4 cases of IVb and 2 cases of IVc were non malignant on histopathological examination.

These lesions were ranging from lcase of high grade DCIS, atypical ductal hyperplasia(3cases), FCC with Usual Ductal Hyperplasia (4 cases), Adenomatous hyperplasia/ Epitheliosis/ Benign epithelial proliferation (4cases), duct papilloma(2cases), l case of tubular adenoma, l case of periductal adenoma, 2 cases of mastitis .(Ref Table 3 )
Table 3: showing distribution of BIRADS Cat IV lesions, based on histopathological findings.

\begin{tabular}{|l|l|l|l|}
\hline Category & $\begin{array}{l}\text { Number } \\
\text { of cases }\end{array}$ & $\begin{array}{l}\text { Discordant Histopathology } \\
\text { findings }\end{array}$ \\
\hline BIRAD & 37 cases & 6 & Adenomatous hyperplasia-2 cases \\
\cline { 3 - 4 } cat IV & & & ADH-2 cases \\
\cline { 3 - 4 } & & & DCIS-2 cases \\
\hline IV a & 10 cases & 6 & Fibrocystic change-4 cases \\
\cline { 3 - 4 } & & & Epitheliosis-2 cases \\
\hline IV b & 5 cases & 4 & Duct papilloma-2 cases \\
\cline { 3 - 4 } & & & Mastitis-2 cases \\
\hline IV c & 12 cases & 2 & Tubular adenoma-l case \\
\cline { 3 - 4 } & & & Periductal adenoma-1 case \\
\hline Total & 64 & 18 \\
\hline
\end{tabular}

BIRADS Category III lesions:

Out of a total of 16 cases (64\%) of BIRADS 3 lesions, ie, probably benign category, $87.5 \%$ of cases showed various benign breast lesions, including 10 cases of fibrocystic change with adenosis, fibrosis and 2 cases each of fat necrosis and fibrocystic change with usual ductal hyperplasia and foci with apocrine change and one case showed only cores of calcified tissue, on histopathological examination and $l$ case each with features of invasive carcinoma of breastmucinous type and early DCIS changes.(Ref Table 4)

Table 4: showing distribution of BIRADS Cat III lesions, based on histopathological findings

\begin{tabular}{|l|l|l|}
\hline Category & $\begin{array}{l}\text { Benign concordant } \\
\text { cases }\end{array}$ & $\begin{array}{l}\text { Discordant malignant } \\
\text { cases }\end{array}$ \\
\hline BIRADS III & $\begin{array}{l}\text { Fibrocystic change- 10 } \\
\text { cases }\end{array}$ & $\begin{array}{l}\text { Mucinous carcinoma- 1 } \\
\text { case }\end{array}$ \\
\cline { 2 - 3 } & Fat necrosis-2 cases & Early DCIS- 1 case \\
\cline { 2 - 3 } & UDH-2 cases & \\
\hline Total cases & 14 & 2 \\
\hline
\end{tabular}

\section{BIRADS Category II lesions:}

Both the cases of BIRADS category II, showed features of fibroepithelial lesion of breast(Fibroadenoma) on histopathological examination.

\section{DISCUSSION}

This is a retrospective study of , histopathological analysis of various BIRADs categories of breast lesions.

A total of 252 patients with various BIRADs category lesions i.e, category II- 2 cases $(0.8 \%)$, category III- 16 cases $(6.5 \%)$, category IV-64 cases (25.4\%) and category V-170 cases $(67.4 \%$ ) were analysed were analysed based on histopathological features In our study, we observed88\% radiologic-pathologic concordance.

Michael et al and O Idowu et al9 in their study found the rate of radiologic"pathologic concordance was as high as $94.5 \%$. [4].

The age group in the present study ranged from 26 years to 89 years, with a mean age group of 53.6 years. Maximum number of cases were in 5th and 4th decade with $28 \%$ and $25 \%$, respectively.

Age is also an important factor in the management of breast lesions. The risk of breast cancer increases with age.[5].

In a study by Nizamettin et al [6], 85.7\% of malignant cases were in postmenopausal period, whereas $14.3 \%$ of them were in premenopausal period. Malignancy rate generally increased in patients over the age of $47 \mathrm{yrs}$.

On histopathological examination,

BIRADs category V: Out of the total 252 cases, category V lesions were $170(67.4 \%)$ cases. 
Out of which , 165 cases show features of carcinoma of breast on histopathological examination, which shows a positive predictive percentage of malignancy as $97 \%$. [Fig l,2]

Nizamettin et al (2019) reported $96.43 \%$ concordance in BIRADS V lesions. [6].
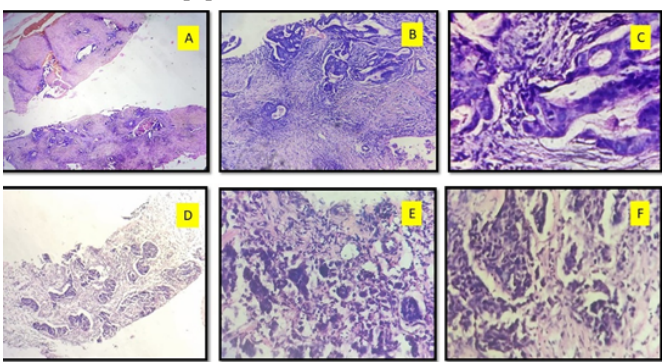

Fig 1: BIRADS V Lesions: A-H\&E $\mathrm{x} 40$ shows cores of tumor tissue, B-H\&E $\mathrm{xl} 00$ shows invasive carcinoma of breast- NST, SBR score 8/9 Grade III, with dense tumor infiltrating lymphocytes, C-H\&E x400, High Grade invasive carcinoma of Breast-NST. D-H\&Ex40 shows core of tumor tissue, E-H\&E x100 shows features of invasive carcinoma of breast, nests and with dense tumor infiltrating lymphocytes, F-H\&E x400- SBR score 7/9, Grade II Invasive carcinoma of Breast.
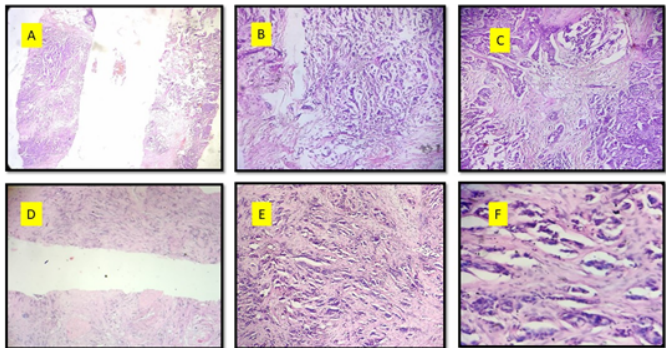

Fig 2:BIRADS V lesions: A-H\&E x40 cores of tumor tissue, BH\&E x100 shows infiltrating cords of tumor cells with desmoplasia, C-H\&Ex400 shows invasive carcinoma of breast-NST with SBR score 7/9, Grade II.

D-H\&E $x 40$ cores of tumor tissue, E-H\&E $x 100$ shows tumor with infiltrating cords of tumor cells with desmoplasia, F-H\&E x400 shows invasive carcinoma of Breast-NST with SBR score 7/9, Grade II.

Only 5 cases of BIRADS V category showed discordance with histopathology, which include 3 cases of duct papilloma [Fig 3], l case of phyllodes tumour and l case of granulomatous mastitis.

In a study by Xiaoni Li et al(2020) [7], 98.99\% of duct papillomas had ultrasound abnormalities, and $53.06 \%$ had intraductal hypoechogenicity upon ultrasound. $31.89 \%$ had mammographic distortion, and $14.45 \%$ had microcalcification upon mammography and therefore may cause discordance with histopathological findings.

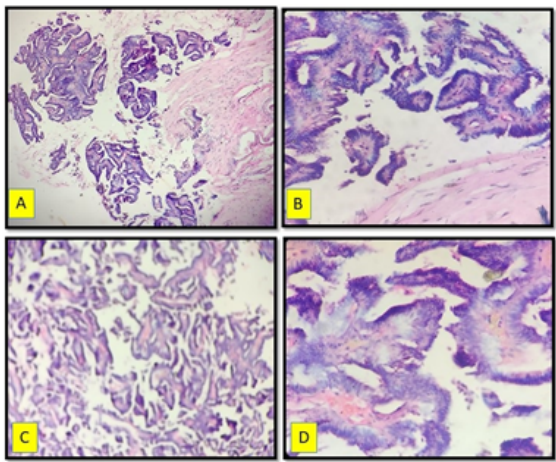

Fig 3: A-H\&E X40 Intraductal Papilloma of breast, B-H\&E x100
Intraductal papilloma of breast, C-H\&E x40 Intraductal Papilloma showing complex papillary branching pattern with fibrovascular cores, D-H\&Ex400 Papilloma showing fibrovascular cores lined by benign duct epithelial cells.

Phyllodes tumor is a rare fibroepithelial tumor of breast, typically seen as non-specific large rounded oval or lobulated, well circumscribed, lesions with smooth margins on mammography. A radiolucent halo may be present and general sonographic features are non-specific and therefore cause discordant histopathological features. [8]

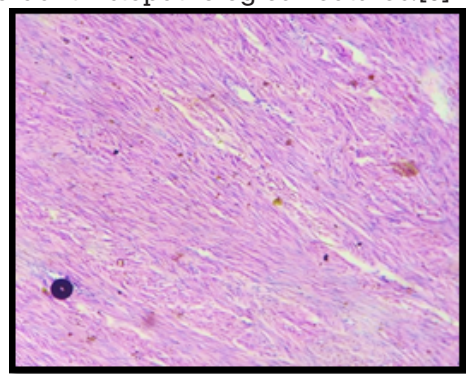

Fig 4: BIRADS V lesion: H\&E, xl00 shows a cellular stromal tumor, with lack of ducts- Borderline phyllodes tumor.

Granulomatous mastitis can mimic breast carcinoma clinically and mammographically. But, sonographically may suggest the possibility of granulomatous mastitis and confirmed by histopathological examination.[9]

Vinod and Ravi Kumar et $a l^{10}$ showed that the concordance rate between the radiologic findings and the histopathology findings was $91.6 \%$ and clearly demonstrated the detection rate of carcinoma was $86 \%$ by Ultrasound.[10]

\section{BIRADs category IV:}

Out of the total 64 cases of BIRADS category IV, only 27 were subcategorised, including 10 cases of $4 a, 5$ cases of $4 b$ and 12 cases of $4 \mathrm{c}$

In our study the rate of malignancy for BIRADS IV lesions was $71.87 \%$ as a whole, $40 \%$ of Iva lesions, $20 \%$ of IVb lesions and $83.4 \%$ of IVC lesions shoed features consistent with malignancy on histopathological examination, including 1 case each of mucinous carcinoma and poorly differentiated carcinoma.

In a study by Nizamettin et al, the malignancy rate was $2.28 \%$ in BIRADS $4 a, 2.86 \% 4 \mathrm{~b}$ and $63.64 \%$ in $4 \mathrm{c}$ lesions.[6]

Histopathological analysis of the discordant BIRADS Category IV cases showed, features, ranging from high grade DCIS(l case), atypical ductal hyperplasia(3cases), FCC with Usual Ductal Hyperplasia (4 cases), Adenomatous hyperplasia, Epitheliosis and Benign epithelial proliferation(4cases), duct papilloma(2cases), l case of tubular adenoma, 1 case of periductal adenoma, 2 cases of mastitis .
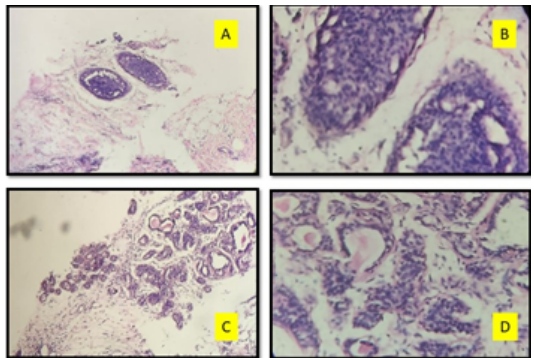

Fig 5: BIRADS IV lesions with discordant histopathological findings. A- H\&E, $\mathrm{x} 40$, cores of breast tissue with epitheliosis/ UDH features, B-H\&E x100 UDH, C- H\&E, $x 40$ Cores of breast 
tissue with focal adenosis. D-H\&E, xl00-showing adenosis.

In the broad category of BIRADS IV, 37 cases concordant cases include malignant lesions like invasive duct cell carcinoma- NST and discordant cases include fibrocystic disease, fibroadenoma, tubular adenoma, intraductal papilloma, phyllodes tumour.

In the subcategories of BIRADS IV lesions, out of 10 cases of $4 \mathrm{~A}, 6$ cases show discordance include cystic neutrophilic granulomatous mastitis, usual ductal hyperplasia, inflammatory lesion and 2 cases show only fibrofatty tissue with no evidence of malignancy.

Out of 5 cases assigned 4B, 2 cases show discordance include ductectasia and atypical ductal hyperplasia.

Out of 12 cases assigned 4C, 8 cases show concordance and 4 cases show discordance.

In BIRADS category IV lesions the concordance rate with histopathological findings increased when they were subcategorised as A, B and C.[1 1].

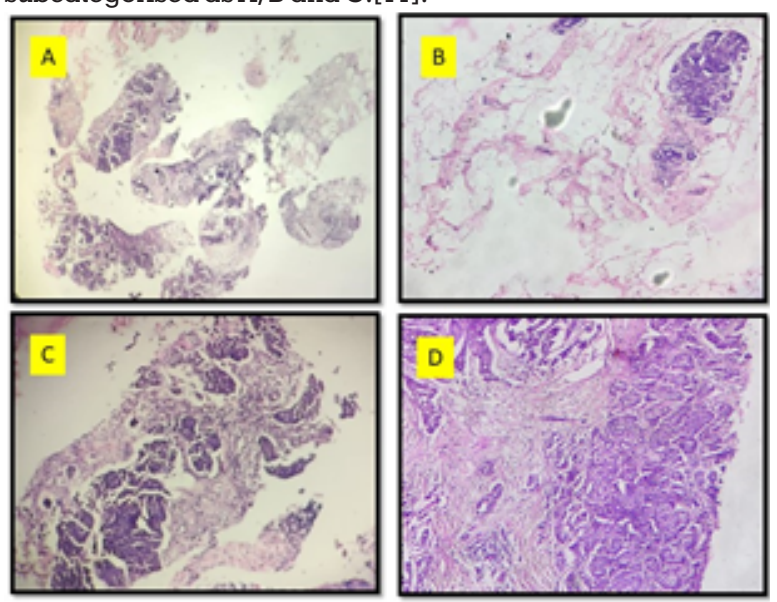

Fig 6:BIRADS IV c lesions: A: H\& E, $\mathrm{x} 40$ shows cores of breast tissue, B: H\&E xl00 shows one pole of the tissue core showing malignant cell clusters, C-H\&E xl00 shows core of tumor tissue with nests and cords of tumor cells, D-H\&E xl00 shows features of an invasive carcinoma of breast-NST.

Whereas, Lee et al reported that PPV for sonography was 26\%, $83 \%$, and $91 \%$ for category $4 a, 4 b$, and $4 c$, respectively.

BIRADs category III: In our study, $87.5 \%$ of BIRADS III lesions showed benign features on histopathological examination, including fibrosing adenosis, intraductal papilloma, fibrocystic disease, fibroadenoma, mastitis and cores of dystrophic calcifications in one case.

The remaining 2 cases (\%) were 1 case each of invasive carcinoma breast-mucinous type and Early DCIS.
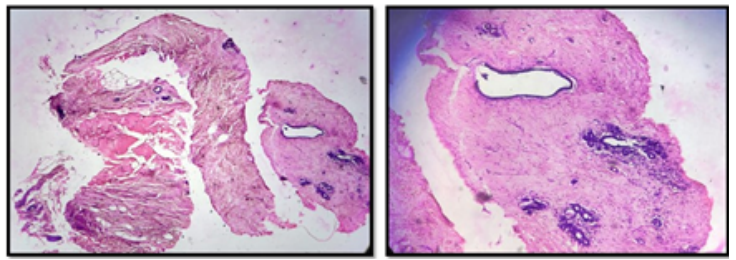

Fig 7: BIRADS III Lesion: H\&E showing cores of breast tissue with features of fibrocystic change of breast, with fibrosis and focal adenosis.

In a study by Nizammettin et al, the malignancy rate was $0 \%$ in BIRADS-3 lesions.[6]
Mentes et al determined the probability of malignancy in BIRADS- 3 as $1.5 \%$ and $32.6 \%$ in BIRADS- 4 lesions.

False-negative diagnosis occurs when the lesion may not have been sampled adequately. Appropriate follow-up imaging is invaluable; even patients with concordant benign findings after US-guided core needle biopsy. [12] A review reported that patients classified as BIRADS-3 have low risk for cancer and 6-month mammography follow-up is appropriate.[13]

Subtle suspicious sonographic features are sometimes overlooked because the final assessment category is determined based on the individual radiologist's experience and training as well as published criteria.

BIRADs category II: Only 2 cases categorised as BIRADs II lesions demonstrated benign pathology with features of a fibroepithelial lesions at core biopsy (fig ).

Many studies reported 100\% concordance of BIRADS II lesion with histopathological examination.

Crystal P et al(2005) concluded that CNB is a safe and accurate method for evaluating breast lesions that require tissue sampling. Radiologic-pathologic correlation and follow-up of benign lesions are essential for a successful breast biopsy program.[14]

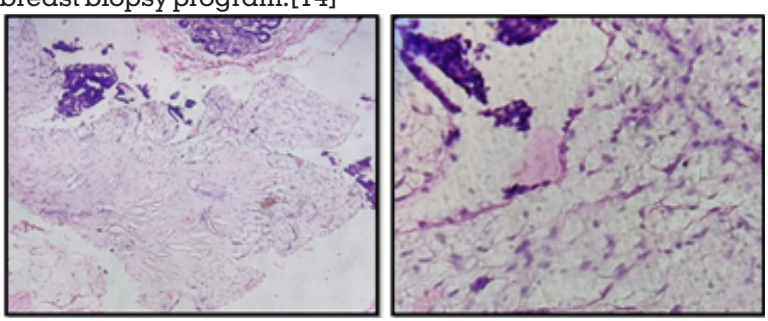

Fig 8: H\&E, $\mathrm{x} 40$ and $\mathrm{xl00}$ shows cores of a fibroepithelial lesion-fibroadenoma of breast with loose myxoid stroma.

\section{CONCLUSION}

Our study 88\% BIRADS lesions showed concordance with histopathological findings. BIRADs category V: a positive predictive percentage of malignancy as $97 \%$, is observed.

A new recommendation for category IV to be subdivided into 3 subgroups (IVa, IVb and IVc) increases the positive predictive value, ie concordant malignant lesions. BIRADs III lesions have lower probability of malignancy. Concordant benign lesions were $81 \%$ and discordant malignant lesions were $19 \%$.BIRADs category II showed $100 \%$ concordance with histopathological findings in the present study. Radiologicpathologic correlation and follow-up of benign lesions are essential for a successful breast biopsy program

\section{REFERENCES}

1. American College of Radiology. Breast Imaging Reporting and Data System (BI-RADS $\left.{ }^{8}\right)$. 4th ed. Reston, VA. American College of Radiology; 2003.

2. American College of Radiology. American College of Radiology Breas Imaging Reporting and Data System BI-RADS, 5th ed, D'Orsi CJ, Sickles EA Mendelso n EB, Morris EA, et al. (Eds), American College of Radiology, Reston, VA 2013

3. Yoon JH, Kim MJ, Moon HJ, Kwak JY, Kim EK. Subcategorization of ultrasonographic BI-RADS category 4: positive predictive value and clinical factors affecting it. Ultrasound Med Biol. 2011;37:693-699.

4. Michael O Idowu, Linday Bonnee Hardy, Rhone J Souers et al. Pathologic diagnostic correlation with Breast imaging Analysis: A college of American Pathologists Q-probes study of 48 Institutes. Arch Pathol Lab Med(2012) 136 (1): 53-60.

5. Smith SA, Saslow D, Sawyer D, et al. American Cancer Society guidelines for Breast Cancer Screening Updat 2003. Ca Cancer J Clin 2003;53: 141-69.

6. Nizamettin Kutluer, AliAksu, Mehmet Burga Bozan, Burhan Hakan Kanat, Hulagu Kargici, Ferhat Cay, Ayse Azak Bozan. Correlation between histopathological results and BI-RADS classification in breast masses. Annals of Medical Research, 2019;26(11):2698-701.

7. Li X, Wang H, Sun Z, Fan C, Jin F, Mao X. A retrospective observational study of intraductal breast papilloma and its coexisting lesions: A real-world 
experience. Cancer Med. 2020 Oct;9(20):7751-7762.

8. Megan Kalambol, Beatriz E. Adradal, Modupe M. Adeyefa2, Savitri Krishnamurthy3 Phyllodes Tumor of the Breast: Ultrasound-Pathology Correlation. American Journal of Roentgenology. 2018;210: W173-W179. 10.2214/AJR.17.18554.

9. Han BK, Choe YH, Park JM, Moon WK, Ko YH, Yang JH, et al. Granulomatous mastitis: mammographic and sonographic appearances. AJR Am J Roentgenol. 1999;173:317-320.

10. Vinod Kumar B, Ravi Kumar A. Ultrasound evaluation of breast masses and histopathology correlation. Int J Contemporary Med Surg Radiol. 2018;3(2):B85-B88.

11. Sweeney DJ, Wylie EJ. Mammographic appearances of mammary duct ectasia that mimic carcinoma in a screening programme. Australas Radiol. 1995;39:18-23.

12. Youk JH, Kim EK, Kim MJ, Lee JY, Oh KK Missed breast cancers at US-guided core needle biopsy: how to reduce them. .Radiographics. 2007 JanFeb;27(1):79-94.

13. Levy L, Suissa M, Teman G et al. BIRADS Ultrasonography. EJ of Radiology 2007:61:202-11.

14. Crystal P, Koretz M, Shcharynsky S, Makarov V, Strano S. Accuracy of sonographically guided 14-gauge core-needle biopsy: results of 715 consecutive breast biopsies with at least two-year follow-up of benign lesions. J Clin Ultrasound. 2005;33:47-52.

15. Schnitt SJ, Collins LC, Biopsy Interpretation of the Breast.lst ed.Newyork: Lippincott Williams \& Wilkins;2009.

16. Park VY, Kim EK, Moon HJ, Yoon JH, Kim MJ. Evaluating imaging-pathology concordance and discordance after ultrasound-guided breast biopsy. Ultrasonography.2018 Apr;37(2):107-120.

17. Radiological and histological correlation of non-palpable breast lesions in patients submitted to preoperative marking according to BI-RADS classification. Radiol Bras [online]. 2007, vol.40, n.1, pp.9-11. ISSN 16787099 